\title{
Exploration of Private Information Pricing Structure
}

\author{
Wenjun Zhang \\ North China Electric Power University. Baoding Hebei 071003, China \\ 305835338@qq.com
}

\begin{abstract}
In the Risk Function, we use AHP calculating the weights of social popularity of the information owner $\left(s_{1}\right)$, subversion of the image of the information owner $\left(s_{2}\right)$,importance of information domain to other people $\left(s_{3}\right)$ and the extent of damage to the public interest $\left(s_{4}\right)$. Then, in order to ensure the accuracy of the function in the real-world, we use multi-expert determination of the subjective weight based on set valued iteration to calculate the weights during simulation. The effect of this way is equal to the effect of the weights calculated by AHP with hypothesis of rational man. We also think of actual situation of the market, so we add expectation as an exogenous variable into the Pricing Structure. Moreover, we draw potential adverse outcome caused by PI being passed maliciously $\left(s_{5}\right)$ into consideration in the simulation event.
\end{abstract}

Keywords: Private information, Risk function, Pricing structure.

\section{Introduction}

"Ring-ring-ring..." a phone call was received. Picking up the phone, "Mr. XX? This is XX Property Company. We think this may interest..." it said. It was known that the answerer's name, phone number and maybe other private information (PI) had been sold to the company as a kind of special goods.

Some people seem willing to share PI about their personal interactions, relationships, and so on, they do not care, while others hold their privacy in these areas as very important and valuable. Moreover, as time goes on, people's opinion on PI have varied greatly. Nowadays, PI is more and more likely to be a kind of special goods that can be priced and sold, just like the phone answerer we just mentioned above. PI gradually become a special commodity. so, we want to know what will happen when PI is sold as a commodity in the market, and we define Risk Function to describe the relationship between the result and the price of special commodity in the paper.

\section{Risk Function}

\subsection{Basic Risk Function}

In order to evaluate the risk of trading PI, we established a risk function. In this part, we only evaluate one single piece of private information. In this function, social popularity of the information owner $\left(s_{1}\right)$, subversion of the image of the information owner $\left(s_{2}\right)$, importance of information domain to other people $\left(s_{3}\right)$ are used to determine how much should the risk of trading private information value.

At last, we can use Analytical Hierarchy Process(AHP) on the 3 indexes above, and come up with 3 weights separately for the 3 indexes, which is named $\alpha_{1}, \alpha_{2}, \alpha_{3}$.Then the final risk value $(R)$ can be calculated with:

$$
R=\boldsymbol{\alpha} \cdot \mathbf{s}^{\mathbf{T}}
$$

Where

$$
\boldsymbol{\alpha}=\left[\alpha_{1}, \alpha_{2}, \alpha_{3}\right], \mathbf{s}=\left[s_{1}, s_{2}, s_{3}\right]
$$




\subsection{The Relationship between Risk Function and Price Point}

Opportunity cost is the value (not a benefit) of the choice of a best alternative cost while deciding [1]. So, we can consider the price point of protecting PI as the opportunity cost that the one suffers from loss because of refusing to sell private information. In this aspect, we can find:

$$
\text { PricePoint }=R=\boldsymbol{\alpha} \cdot \mathbf{s}^{\mathbf{T}}
$$

\subsection{Risk Function and Price Point under Further Consideration}

We already have a risk function to evaluate risk in simple condition. However, in some other conditions, the indexes needed should be more. For example, in the fields of disease control, or in the field of tracking terrorists, the risk of trading one's PI is additionally influenced by the potential extent of damage to the public interest $\left(s_{4}\right)$. With a weight also gotten through AHP, the new $\boldsymbol{\alpha}$ and $\mathbf{x}$ in (1) should be $\left[\alpha_{1}, \alpha_{2}, \alpha_{3}, \alpha_{4}\right]$ and $\left[s_{1}, s_{2}, s_{3}, s_{4}\right]$.

Because of the hypothesis of rational man and second sale is prohibited, we can ignore the potential bad result happen to the owner of selling PI.

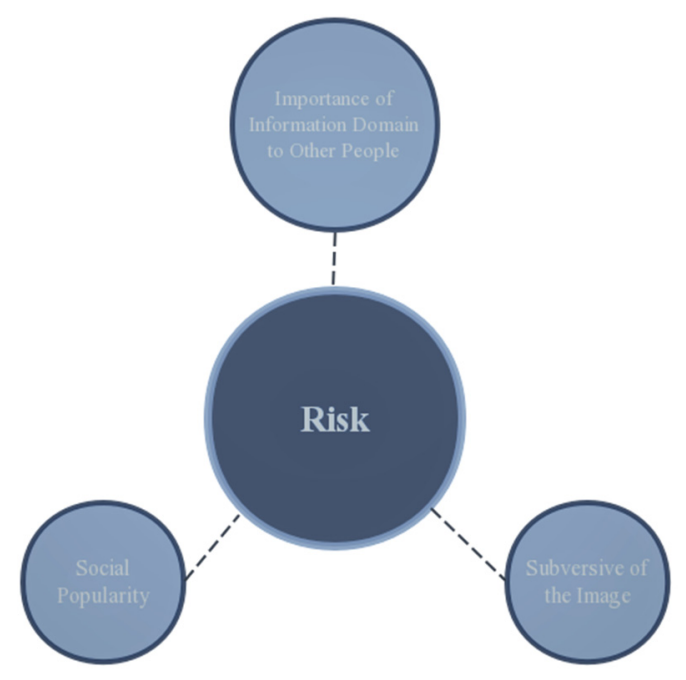

Fig. 1 Three elements

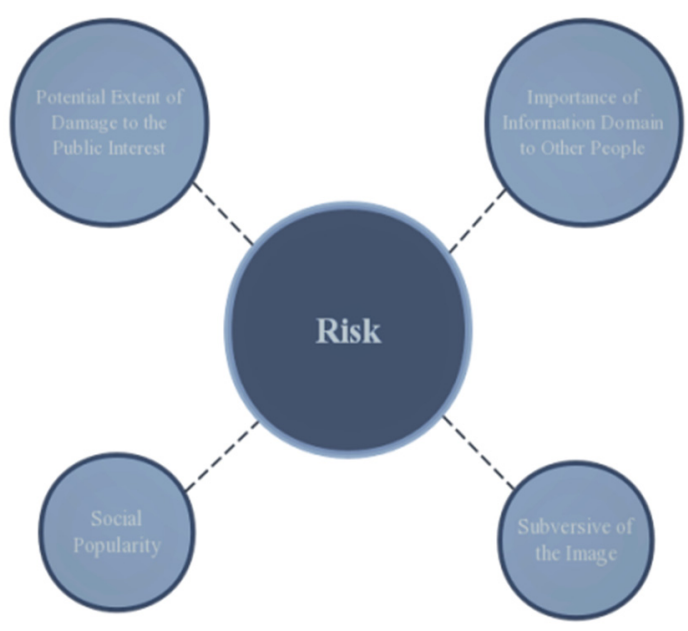

Fig. 2 Four elements

\subsection{Pricing Structure under Consideration of Perception}

We study how the age of PI owner impact the pricing structure, and find that people of different ages generally have different perception on each $\alpha$ in the risk Function. We can describe the influences caused by age vary with a series function $f_{i}(A)$, where $A$ is on behalf of the owner's age, $f_{i}(A)$ is on behalf of the influence extent on $\alpha_{i}$. So, in the new pricing structure, which put agerelated perception differences into consideration, the weights in the risk Function should be renewed as

$$
\alpha_{i}{ }^{\prime}=f_{i} \cdot \alpha_{i}
$$

So, the risk should be calculated with

$$
R=\boldsymbol{\alpha}^{\prime} \cdot \mathbf{s}^{\mathbf{T}}
$$

Where 


$$
\boldsymbol{\alpha}^{\prime}=\left[\alpha_{1}{ }^{\prime}, \alpha_{2}{ }^{\prime}, \alpha_{3}{ }^{\prime}\right]
$$

The pricing structure should be dependent on the renewed risk Function.

We found a survey that reveals the influence of age on perception of PI. The figures are as follows:

Table 1. People of different ages' Acknowledgement of PI Importance [2].

\begin{tabular}{cc|cccccc}
\hline Age & Number & Name & Age & Tel & Medical Examination Report & Past History & Social Relations \\
\hline$<30$ & 41 & $29.27 \%$ & $24.39 \%$ & $31.71 \%$ & $51.22 \%$ & $43.90 \%$ & $19.51 \%$ \\
$30 \sim 39$ & 49 & $65.31 \%$ & $71.43 \%$ & $59.18 \%$ & $63.27 \%$ & $57.14 \%$ & $79.59 \%$ \\
$40 \sim 49$ & 53 & $79.25 \%$ & $96.23 \%$ & $81.13 \%$ & $94.34 \%$ & $88.68 \%$ & $92.45 \%$ \\
$50 \sim 59$ & 51 & $76.47 \%$ & $98.04 \%$ & $80.39 \%$ & $96.08)$ & $94.12 \%$ & $92.16 \%$ \\
$>=60$ & 40 & $22.50 \%$ & $130.00 \%$ & $15.00 \%$ & $42.50 \%$ & $27.50 \%$ & $7.50 \%$ \\
\hline
\end{tabular}

So, we can come up with the following result:

Table 2. Result [2]

\begin{tabular}{cc|ccc}
\hline Age & Number & Very Important & Important & Not important \\
\hline$<30$ & 41 & $15(36.59 \%)$ & $17(41.46 \%)$ & $9(21.95 \%)$ \\
$30 \sim 39$ & 49 & $32(65.31 \%)$ & $10(20.41 \%)$ & $7(14.28 \%)$ \\
$40 \sim 49$ & 53 & $43(81.13 \%)$ & $5(9.43 \%)$ & $5(9.43 \%)$ \\
$50 \sim 59$ & 51 & $42(82.35 \%)$ & $6(11.76 \%)$ & $3(5.88 \%)$ \\
$>=60$ & 40 & $13(32.50 \%)$ & $9(22.50 \%)$ & $18(45.00 \%)$ \\
\hline
\end{tabular}

We can truly see the differences between subjects of different ages in the survey. People of the age 40 49 and 50 59 aged people evaluate their PI highly. That is because majority of these people have had their own career and social reputation and worry to be influenced by the PI breach risk, which make them evaluate their PI higher. While the $<30$ aged people are usually naive youngers, who do not think so highly of PI and the $>=60$ aged people are mainly retired old people, who do not think so highly of career and reputation like before, that is why they don't evaluate their PI as highly as the middle-aged people.

\subsection{Simulation: A Massive Data Breach}

The function is based on the hypothesis of rational man. However, the rational man does not exist in reality. so, we shall use multi-expert determination of the subjective weight based on set valued iteration [2], with a huge number of subjects determining. This method can make the evaluation more accurate than what AHP can make.

If millions of people's PI are somehow stolen and sold on a dark web, which is not allowed in the assumptions above, the evaluation of risk should be renewed in two aspects. The first is the potential adverse outcome caused by PI being passed maliciously $\left(s_{5}\right)$, and the second is people may reconsider the evaluating of $\alpha$ because of the change of their attitude to PI sale. Each of the two aspects can lead to an increase of the value of $R$. An example of PI loss influencing risk evaluating is the big reduction of iPhone selling caused by the PRISM [3]. 


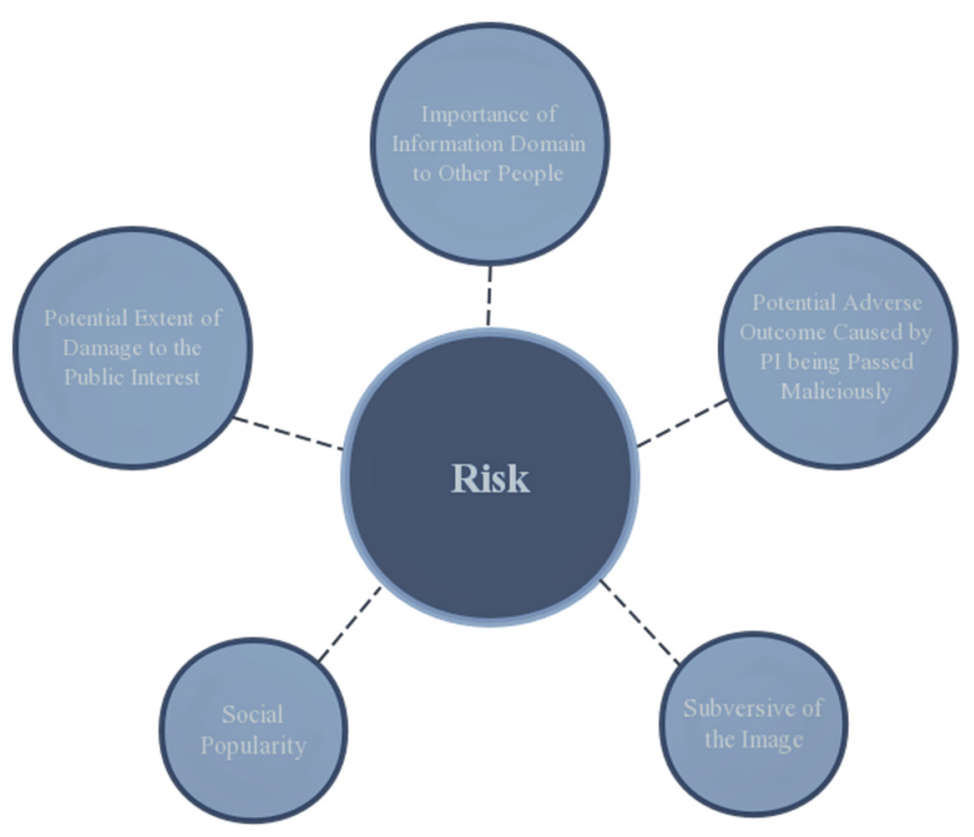

Fig 3. Five elements

\section{Conclusion}

In the paper, we define Risk Function.We think all of risks can be changed into economy cost, so we present the relationship between it and the price of special commodity under this assumption.

In this function, social popularity of the information owner $\left(s_{1}\right)$, subversion of the image of the information owner $\left(s_{2}\right)$, importance of information domain to other people $\left(s_{3}\right)$ are used to determine how much should the risk of trading private information value.Meanwhile, we present the price of special commodity accordinging to the function. Further, we think the risk of trading one's PI is additionally influenced by the potential extent of damage to the public interest $\left(s_{4}\right)$,so we supplement the Risk Function and Pricing Structure.

We think of actual situation of the market, so we add expectation as an exogenous variable into the Pricing Structure. Through the Wu Ying's survey, we know the views of different age groups on selling personal information, and draw the conclusion that this variable will change with age.

At last, we also make the simulation under the Massive Data Breach to prove feasibility of our function.

\section{References}

[1]. Opportunity cost. https: //en. wikipedia. org/ wiki/Opportunity_cost.

[2]. Li De Shun, Xu Kai Li, Li Chun Fu. Multi-Expert Determination of the Subjective Weight Based on Set Valued Iteration[J].2009.

[3]. Effect of PRISM of Apple's mobile phone market.http://www.admin5.com/article/20140807/55 5640.shtml. 